\title{
Inversão uterina não puerperal em paciente jovem por mioma parido gigante: relato de caso e revisão de literatura
}

\section{Non puerperal uterine inversion in a young patient for a giant paritus leiomyoma: case report and review}

\author{
Emilcy Rebouças Gonçalves ${ }^{1}$. Leonardo Robson Pinheiro Sobreira Bezerra² ${ }^{2}$ Sara Arcanjo Lino Karbage ${ }^{3}$. \\ Alan Prado Rocha ${ }^{4}$. \\ 1 Residente do $3^{\circ}$ ano em Ginecologia e Obstetrícia, Maternidade Escola Assis Chateaubriand (MEAC), Universidade Federal \\ do Ceará (UFC), Fortaleza, Ceará, Brasil. 2 Doutor em Ciências pela Universidade Federal de São Paulo (UNIFESP), Professor \\ Adjunto do Departamento de Saúde Materno Infantil da Universidade Federal do Ceará (UFC), Fortaleza, Ceará, Brasil. 3 \\ Mestre em saúde Coletiva pela Universidade de Fortaleza (UNIFOR), Médica Ginecologista-Obstetra da Maternidade Escola \\ Assis Chateaubriand (MEAC), Universidade Federal do Ceará (UFC) - Empresa Brasileira de Serviços Hospitalares (EBSERH), \\ Fortaleza, Ceará, Brasil. 4 Médico Ginecologista-Obstetra da Maternidade Escola Assis Chateaubriand (MEAC), Universidade \\ Federal do Ceará (UFC), Fortaleza, Ceará, Brasil.
}

\section{RESUMO}

A inversão uterina não puerperal em paciente com 45 anos ou menos é quadro clínico de rara descrição na literatura e está associado à presença de tumor uterino em mais de $90 \%$ dos casos, sendo o mais comum o leiomioma submucoso. Em revisão de literatura, foram encontrados apenas 23 casos de inversão uterina não puerperal em paciente com 45 anos ou menos, entre 1887 e 2015 . Neste artigo, é relatado o caso de uma mulher de 39 anos, nulípara, admitida em unidade de emergência por queixa de volumoso tumor endurecido e de odor fétido que tinha se exteriorizado agudamente através da fenda vulvar, associada a dor abdominal em hipogástrio e sangramento transvaginal profuso. No intraoperatório, confirmou-se a hipótese de inversão uterina aguda não-puerperal, sendo procedida a exérese da massa tumoral por via vaginal, complementada com histerectomia total por via abdominal e vaginal. A paciente evoluiu bem no pós-operatório, sem quaisquer intercorrências. O exame histopatológico revelou tratar-se de mioma submucoso.

Palavras-chave: Inversão uterina. Leiomioma. Hemorragia uterina.

\section{ABSTRACT}

Non-puerperal uterine inversion in patients aged 45 years or less is a clinical condition rarely described in the literature and associated with uterine tumor by more than $90 \%$ of cases, of which the most common type is the submucosal leiomyoma. In a literature review, it was found only 23 cases of non-puerperal uterine inversion in patients aged 45 years or less, between 1887 and 2015. In this article, we report a case of a 39-years-old woman, nulliparous, who was admitted to the emergency department by a complaint of a hardened and foul odor huge tumor that had been sharply externalized across the vulvar slit, associated with abdominal pain in hypogastrium and profuse transvaginal bleeding. During surgery, it was confirmed the hypothesis of acute non-puerperal uterine inversion, being done resection of the tumor vaginally, complemented with total abdominal and vaginal hysterectomy. The patient recovered well post operatively, without any complications. Histopathological examination revealed submucosal myoma.

Keywords: Uterine inversion. Leiomyoma. Uterine bleeding.

Autor correspondente: Emilcy Rebouças Gonçalves, Rua Rúbia Sampaio, 1219, Farias Brito, Fortaleza, Ceará. CEP: 60011-060.Telefone:. E-mail: emilcyreb@gmail.com

Conflito de interesses: Não há qualquer conflito de interesses por parte de qualquer um dos autores.

Recebido em: 22 Fev 2016; Revisado em: 15 Mar 2016; Aceito em: 22 Mar 2016. 


\section{INTRODUÇÃO}

A inversão uterina é um quadro clínico de rara descrição na literatura. Pode ser classificada em dois tipos: (1) puerperal ou obstétrica e (2) não-puerperal ou ginecológica. ${ }^{1}$ A fisiopatologia da inversão não-puerperal parece ser multifatorial, incluindo três principais fatores etiológicos: ${ }^{2}$ crescimento rápido da neoplasia uterina, adelgaçamento das paredes uterinas pelo tumor intra-uterino e distensão da cavidade levando à dilatação do colo do útero. Em geral, a morbidade e a mortalidade são baixas, porém se correlacionam diretamente com o grau de hemorragia, a demora do diagnóstico e o tratamento ineficaz. ${ }^{3}$ Gomez-Lobo et al. ${ }^{4}$ registrou 150 casos do tipo não-puerperal entre os anos de 1887 a 2006. Conforme Mwinyoglee et al. ${ }^{5}$ documentou em sua revisão, 97,4\% das inversões uterinas são associadas a tumores, dos quais $20 \%$ são malignos. Casos de inversão não-puerperal são menos frequentes em mulheres com 45 anos ou menos. ${ }^{6,7}$ Contudo, este é o quarto caso semelhante descrito em mulheres nesta faixa etária neste serviço. ${ }^{8,9}$

\section{RELATO DE CASO}

Mulher de 39 anos, nulípara, foi admitida em unidade de emergência com queixa clínica de volumosa tumoração endurecida e de odor fétido que havia se exteriorizado através da fenda vulvar há poucas horas (Figura 1). Associado ao quadro havia dor abdominal em hipogástrio do tipo cólica e sangramento transvaginal profuso. A paciente relatou ciclos menstruais regulares, com aumento do fluxo há dois anos, além de progressiva dificuldade de iniciar a micção, nas últimas semanas. À avaliação inicial, encontrava-se consciente, orientada, hipocorada 3+/4 e hemodinamicamente estável. Foram solicitados exames laboratoriais de urgência, que revelaram hemoglobina $6,8 \mathrm{~g} / \mathrm{dL}$ e hematócrito 22,1\%. Após estabilização inicial, expansão volêmica com cristalóides e hemotransfusão, foi indicada intervenção cirúrgica de urgência.

Após o bloqueio anestésico, tentou-se realizar a redução do tumor para a cavidade pélvica, contudo não houve êxito. Após tração da massa com auxílio de pinças de Lahey, iniciou-se a secção, por via vaginal, da tumoração uterina, utilizando bisturi. Após a retirada de aproximadamente $80 \%$ da massa, foi possível confirmar a hipótese de inversão uterina ao visualizar a serosa uterina, conforme visto na Figura 2.

Para melhor definição dos planos de clivagem e inspeção da cavidade abdominal, foi realizada incisão abdominal de Pfannenstiel. O inventário da cavidade revelou a presença de útero aumentado de volume e invertido, pela identificação dos pedículos anexiais e ligamentos redondos afunilados e concêntricos na escavação pélvica. Tentou-se novamente a redução da inversão uterina pela técnica de Johnson e Huntington, sem sucesso.

Após identificação, procedeu-se com secção e ligadura de pedículos ovarianos e ligamentos redondos, seguida de dissecção do espaço vesico-uterino e ligadura das artérias uterinas de modo inverso. Após isso, retornou-se à via vaginal para exérese do útero por morcelamento pela técnica de Lash. A cirurgia foi finalizada com a sutura contínua da cúpula vaginal por via abdominal, com fixação em ligamentos redondos e uterosacrocardinais remanescentes (Figura 3).

Figura 1. Mioma parido gigante em mulher de 39 anos

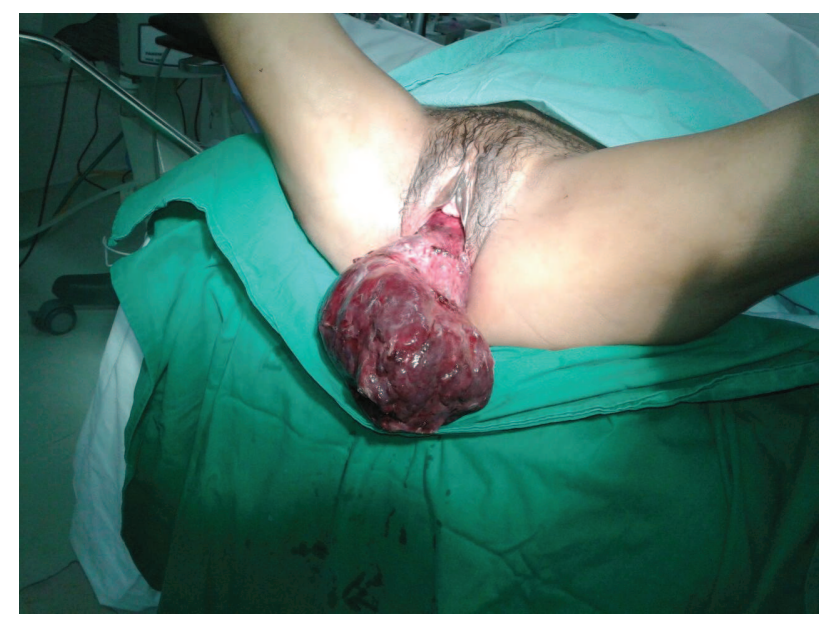

Fonte: elaborada pelos autores.

Figura 2. A: Secção do pedículo da tumoração com bisturi. B: Identificação inicial de serosa uterina ainda durante a secção do pedículo da tumoração. C: Achado intraoperatório de inversão uterina não puerperal, com visualização de serosa uterina e ligamentos redondos (seta).

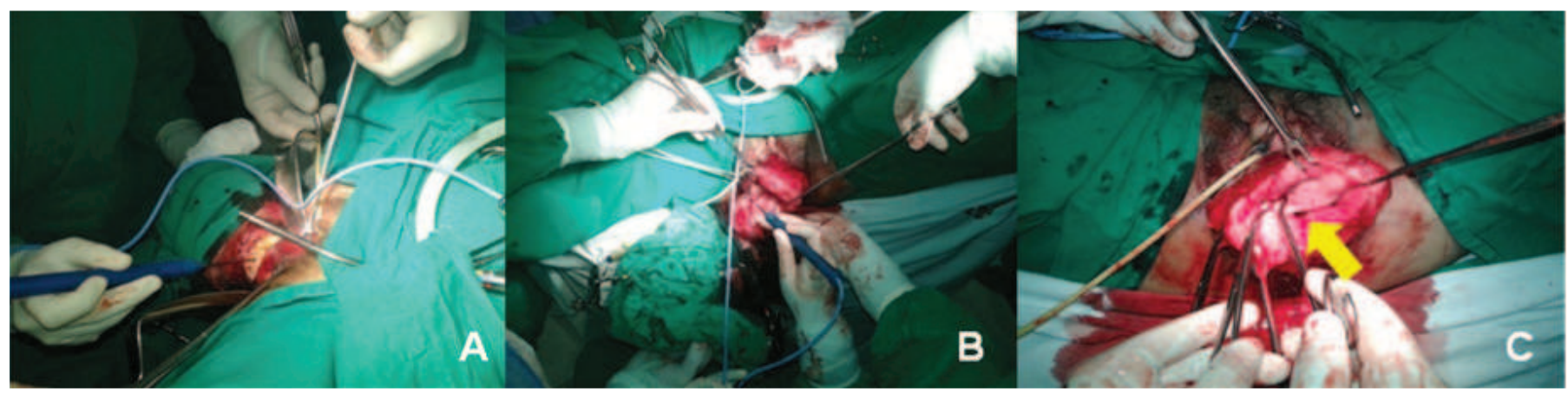

Fonte: elaborada pelos autores. 
Figura 3. A: Sutura da cúpula vagina por via abdominal com pontos contínuos e fixação em ligamentos redondos e uterossacros remanescentes. B: Aspecto final da cúpula vaginal já suturada e fixada. C: Aspecto final da vulva ao final da cirurgia.

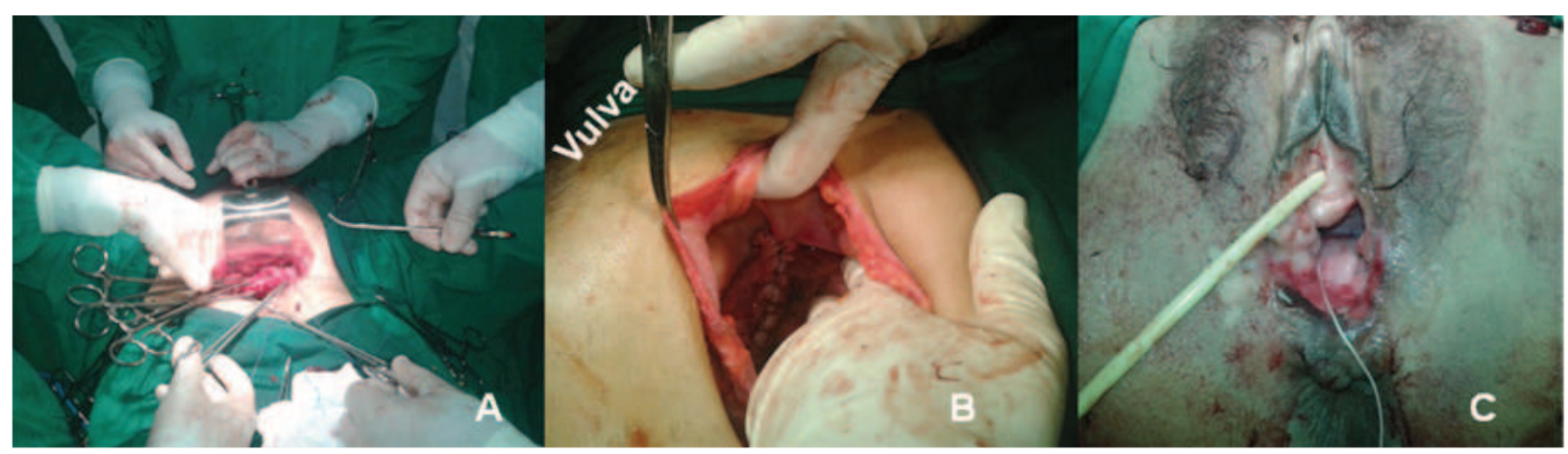

Fonte: elaborada pelos autores.

Foi mantido cobertura antibiótica com clindamicina $600 \mathrm{mg}$ IV de $8 / 8$ horas e gentamicina $240 \mathrm{mg}$ IV de $24 / 24$ horas por 7 dias. A paciente foi de alta hospitalar 8 dias após o procedimento, sem quaisquer intercorrências no pós-operatório.
A análise histopatológica da peça cirúrgica confirmou que se tratava de leiomioma submucoso parido de $17 \times 15 \times 10 \mathrm{~cm}$ e 1200 g (Figura 4). Foi realizada avaliação ambulatorial da paciente após 38 dias de cirurgia, quando ela se mostrou bastante satisfeita com o procedimento.

Figura 4. A: Macroscopia da peça cirúrgica, que mediu 17x15x10 cm e pesou $1200 \mathrm{~g}$. Na seta, parte da parede uterina. B: Microscopia da peça cirúrgica, evidenciando leiomioma com diversos focos hemorrágicos (aumento de 100x).

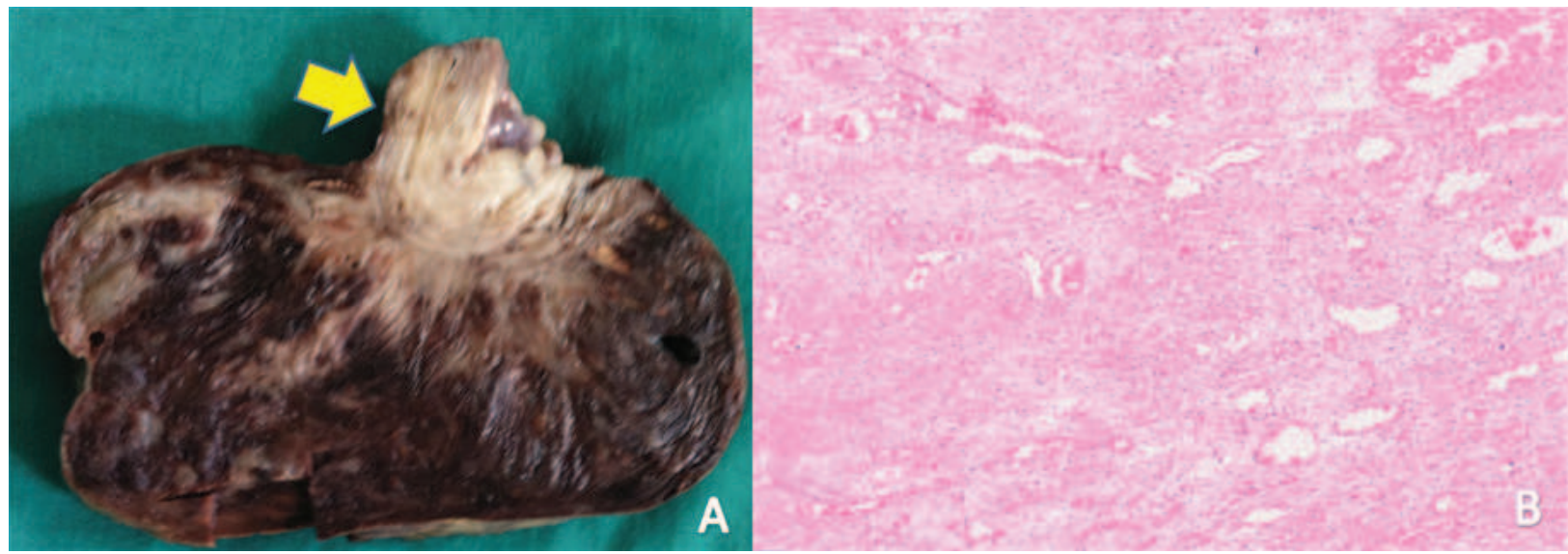

Fonte: elaborada pelos autores.

\section{DISCUSSÃO}

A inversão uterina não puerperal é rara na prática clínica, especialmente em pacientes jovens. Devido a isso, realizamos pesquisa bibliográfica em busca das melhores evidências científicas para responder à pergunta clínica: "Qual é a frequência de casos de inversão uterina não-puerperal em pacientes com 45 anos ou menos e quais são as intervenções mais eficazes para este caso?" Cinco bases de dados foram examinadas: CINAHL, MEDLINE via PubMed, Cochrane Library, Scopus e LILACS, utilizando a palavra-chave "uterine inversion". Os artigos foram incluídos se escritos em inglês, espanhol, francês e português, relatando os relatos de casos e as estratégias de intervenção. Excluímos aqueles nos quais a causa da inversão era puerperal ou obstétrica, e também aqueles que se referiam a pacientes com mais de 45 anos. Destacamos o artigo de Gomez-Lobo et al., que, no período de 1887 a 2006, registrou apenas três casos de mulheres de 45 anos de idade ou menos com diagnóstico de inversão uterina não puerperal. Analisando os trabalhos publicados posteriormente, encontramos outros 20 casos descritos entre 2007 e 2015 (Quadro 1). Desses, 14 tiveram diagnóstico histológico de leiomioma na peça cirúrgica, 05 foram diagnosticados como outros tumores (dois por teratoma imaturo, um por sarcoma de estroma endometrial, um por rabdomiosarcoma e um por cisto mulleriano) e quatro não se encontrou diagnóstico histológico citado no artigo. Dos quatro casos catalogados neste serviço entre 1975 e 2016 , todos encontravam-se nesta faixa etária e tiveram diagnóstico histológico de leiomioma.

Dor pélvica e sangramento vaginal constituíram as queixas clínicas mais frequentemente referidas, ${ }^{1-9}$ assemelhando-se ao que foi descrito nesse relato de caso. Relatou-se por vezes associação a sintomas urinários por provável compressão uretral extrínseca. ${ }^{2,7}$ A presença de massa tumoral exteriorizada pelo orifício cervical também é frequente, contudo, em alguns casos, somente é percebida durante exame físico ${ }^{8}$ A suspeição clínica para o diagnóstico de inversão uterina ocorre se, em exame genital bimanual, não é possível palpar o fundo uterino. A complementação com o toque retal, para confirmar 
Quadro 1. Sumário dos casos de inversão uterina não-puerperal em pacientes com 45 anos ou menos entre 1887 e 2015.

\begin{tabular}{|c|c|c|c|c|c|}
\hline Referência & $\begin{array}{l}\text { No. de } \\
\text { casos }\end{array}$ & Idade & Via Cirúrgica & Técnica/Cirurgia Realizada & Histopatológico \\
\hline Birge e Merdin, 2015 & 1 & 15 & Laparotomia & Haultain, com preservação do útero & Desconhecido \\
\hline Birge et al, 2015 & 1 & 17 & Laparotomia & Haultain, com preservação do útero & Desconhecido \\
\hline Zhang et al, 2015 & 1 & 34 & Lasparoscopia & $\begin{array}{l}\text { Secção de parede anterior, com } \\
\text { preservação do útero }\end{array}$ & Desconhecido \\
\hline Rathod et al, 2014 & 1 & 28 & Vaginal + Laparotomia & Kustner, com preservação do útero & Mioma \\
\hline Shabbir et al, 2014 & 1 & 39 & Vaginal + Laparotomia & Miomectomia vaginal + Haultain + HTA & Mioma \\
\hline Kulkarni e Ajmera, 2014 & 1 & 40 & Vaginal & Miomectomia vaginal + HTV & Mioma \\
\hline Souza et al, 2014 & 1 & 23 & Laparotomia & $\mathrm{HTA}+\mathrm{SOB}+$ esvaziamento & $\begin{array}{l}\text { Teratoma uterino } \\
\text { imaturo }\end{array}$ \\
\hline Shivanagappa et al, 2013 & 1 & 28 & $\begin{array}{l}\text { Laparoscopia diagnóstica, } \\
\text { seguida de abordagem } \\
\text { abdominal + vaginal }\end{array}$ & HTA & Mioma \\
\hline Tibrewal et al, 2012 & 1 & 45 & Vaginal + Laparotomia & $\begin{array}{l}\text { Miomectomia vaginal }+ \text { Haultain }+ \text { com } \\
\text { HTA }\end{array}$ & Mioma \\
\hline Duhan et al, 2013 & 1 & 24 & $\begin{array}{l}\text { Laparoscopia, seguida de } \\
\text { abordagem vaginal }\end{array}$ & $\begin{array}{l}\text { Miomectomia vaginal + Haultain, com } \\
\text { preservação do útero }\end{array}$ & Mioma \\
\hline Atalay et al, 2013 & 1 & 42 & Vaginal & Miomectomia vaginal + HTV & Mioma \\
\hline Bertrand et al, 2011 & 1 & 41 & Desconhecido & Desconhecido & Mioma \\
\hline Zechmeister e Levey, 2011 & 1 & 37 & Laparoscopia robótica & Preservação uterina & Desconhecido \\
\hline Shirota et al, 2011 & 1 & 27 & Vaginal + Laparotomia & $\begin{array}{l}\text { Miomectomia vaginal + Preservação } \\
\text { uterina }\end{array}$ & Mioma \\
\hline Omololu et al, 2011 & 1 & 37 & Vaginal & HTV & Mioma \\
\hline Auber et al, 2011 & 1 & 40 & Vaginal + Laparotomia & Histerectomia laparoscópica & Mioma \\
\hline Fofie e Baffoe, 2010 & 1 & 42 & Vaginal & HTV & Mioma \\
\hline Vries e Perquin, 2010 & 1 & 19 & Vaginal + Laparotomia & $\begin{array}{l}\text { Miomectomia vaginal + laparoscopia } \\
\text { diagnóstica + laparotomia para correção } \\
\text { da rotura uterina, com preservação do } \\
\text { útero }\end{array}$ & Mioma \\
\hline Eigbefoh et al, 2009 & 1 & 38 & Laparotomia & HTA & Mioma \\
\hline Gomez-Lobo et al, 2007 & 1 & 15 & Laparotomia & $\begin{array}{l}\text { Correção da inversão por Huntington e } \\
\text { Haultain, com preservação do útero }\end{array}$ & $\begin{array}{l}\text { Teratoma uterino } \\
\text { imaturo }\end{array}$ \\
\hline Lupovitch et al , 2005 & 1 & 26 & Laparotomia & HTA radical & $\begin{array}{l}\text { Sarcoma de estroma } \\
\text { endometrial }\end{array}$ \\
\hline Case et al, 2005 & 1 & 21 & Desconhecido & Haultain + histerectomia secundária & Rabdomiosarcoma \\
\hline Desconhecida (ano de 1924) & 1 & 15 & Desconhecido & Eversão & Cisto mulleriano \\
\hline TOTAL & 23 & & & & \\
\hline
\end{tabular}

Fonte: elaborado pelos autores.

a ausência de útero na pelve, parece ser útil, ${ }^{8}$ especialmente em casos de inversões mais graves, em que a vagina encontrase ocupada pela tumoração ou pelo útero invertido.

Realização de exames complementares, como ultrassonografia e ressonância nuclear magnética, são relatados na literatura, seja com o intuito de sanar dúvidas acerca do exame físico, seja para auxiliar no planejamento cirúrgico. ${ }^{2}$ A visualização da cavidade uterina em forma de " $U$ " nestes exames sugere bastante o achado de inversão uterina. ${ }^{10}$ No caso desta paciente relatada, a necessidade de intervenção cirúrgica imediata minimizou a necessidade de avaliação complementar, uma vez que a resolução cirúrgica se tornou preponderante.

Ainda permanece controverso o tratamento cirúrgico mais adequado para inversão uterina não-puerperal. Os artigos 
publicados até o momento não descrevem protocolos clínicos padronizados ou ensaios randomizados com as melhores evidências científicas sobre a conduta mais adequada, pois consistem, na maioria, de relatos de caso. Nesta revisão, observamos frequência maior de intervenções cirúrgicas realizadas por via abdominal, aliadas ou não a outras vias, como a laparoscópica e a vaginal. Observa-se que são poucas as situações onde apenas uma via cirúrgica é utilizada, provavelmente devido à distorção anatômica, que exige do cirurgião a capacidade de se adaptar às peculiaridades de cada caso. Uma vez que não há padronização ou consenso sobre a técnica ideal, as técnicas descritas são muitas. Quando se opta pela via vaginal, Johnson propõe a elevação do útero de maneira forçada até a cicatriz umbilical, para provocar a distensão dos ligamentos e fazer com que o anel cervical se amplie. ${ }^{11}$ Já Spinelli propõe a colpohisterotomia anterior mediana, após a dissecção do espaço vesico-uterino, para reversão do anel cervical até o corpo uterino, procedendose à reversão e à sutura da parede uterina, ou histerectomia vaginal. ${ }^{11}$ Para Küstner, a abordagem proposta é pelo saco posterior, com uma incisão cervical semelhante a partir do anel cervical para o corpo uterino. ${ }^{12}$

Ao se optar pela via abdominal, a redução da inversão uterina é possível pela técnica de Haultain, cujos passos são semelhantes à de Küstner, só que através de laparotomia. Já Huntington propôs a tração progressiva sobre os ligamentos redondos utilizando pinças Allis, também por via abdominal. ${ }^{11,12}$

\section{REFERÊNCIAS}

1. Jones, HW. Non-puerperal inversion of the uterus. Am J Surg [Internet]. 2015 [acesso em 20 dez. 2015];81(5):492-5. Disponível em: http://api.elsevier.com/content/article/PII:0002961051902681?h ttpAccept=text/xml

2. Ishida H, Yano T, Yasuda Y, Takashima A, Takeshita N, Kinoshita T. Nonpuerperal uterine inversion due to submucous leiomyoma. Clin Pract. 2011;1(4):e105.

3. Ashraf-Ganjooie T. Non-puerperal uterine inversion: a case report. Arch Iranian Med. 2005;8(1):63-6.

4. Gomez-Lobo V, Burch W, Khanna PC. Nonpuerperal uterine inversion associated with an immature teratoma of the uterus in an adolescent. Obstet Gynecol. 2007;110(2 Pt 2):491-3.

5. Mwinyoglee J, Simelela N, Marivate M. Non-puerperal uterine inversions. A two case report and review of literature. Cent Afr J Med. 1997;43(9):268-71.

6. Pelissier-Komorek A, Lucereau-Barbier M, Diab J, Gavillon N, Graesslin O. Acute non-puerperal uterine inversion the third degree. Gynecol Obstet Fertil. 2013;41(2):130-2.

7. Casanova J, Huang K, Adlan A, Artazcoz S. Uterine inversion caused
As vias que oferecem abordagem minimamente invasiva, como a laparoscópica e a robótica, vem sendo cada vez mais utilizadas, principalmente em associação com a via vaginal. ${ }^{13,14}$ Entretanto, faz-se necessário a disponibilidade de material específico e de equipe profissional adequadamente treinada para execução dessas técnicas, o que ainda traz limitação ao seu uso, principalmente em casos que demandam abordagem cirúrgica de urgência.

O caso aqui relatado foi então resolvido com a realização de miomectomia por via vaginal, seguida por histerectomia por vias abdominal e vaginal. É relevante salientar que foi optado por realizar uma cirurgia não-conservadora devido ao avançado estágio de necrose do tumor, associado à dificuldade para vencer o anel fibroso ao redor do colo e ao volumoso sangramento de intraoperatório, ainda que a paciente fosse nulípara.

O uso de antibióticos no pós-operatório é controverso, e não há consenso sobre o tipo de antibiótico e tempo de uso mais adequados. Observou-se, na revisão de literatura, que na maioria das vezes não há descrição de tal uso, ou quando utilizado, está associado à presença de febre ou infecção confirmadas. Neste caso, julgou-se prudente o uso do antibiótico pelo achado de extensa necrose tumoral.

by a submucous leiomyoma. J Gynecol Surg. 2013;29(6):294-6.

8. Pinheiro LS, Carvalho AA, Araújo JG. Inversão uterina ginecológica crônica. J Bras Ginec. 1980;89(5):297-300.

9. Pinheiro LS, Ponte JG. Acute nonpuerperal uterine inversion. Int Surg. 1975;60(10):559-60.

10. Hu CF, Lin H. Ultrasound diagnosis of complete uterine inversion in a nulliparous woman. Acta Obstet Gynecol Scand. 2012;91(3):37981 .

11. Neves J, Cardoso E, Araújo C, Santo S, Gonçalves P, Melo A, et al. [Uterine inversion]. Acta Med Port. 2006;19(2):181-4.

12. Lascarides E, Cohen M. Surgical management of nonpuerperal inversion of the uterus. Obstet Gynecol. 1968;22(3):376-381.

13. Auber M, Darwish B, Lefebure A, Ness J, Roman H.. Management of nonpuerperal uterine inversion using a combined laparoscopic and vaginal approach. Am J Obstet Gynecol. 2011;204(6):e7-9.

14. Zechmeister JR, Levey KA. Successful robotically assisted laparoscopic correction of chronic uterine inversion. J Minim Invasive Gynecol. 2011;18(5):671-3

\section{Como citar:}

Gonçalves ER, Bezerra LR, Karbage SA, Rocha AP. Inversão uterina não puerperal em paciente jovem por mioma parido gigante: relato de caso e revisão de literatura. Rev Med UFC. 2016 jul-dez;56(2):58-62. 